\title{
Design and Research of Automatic Smart Waste Container Bag Changing Device
}

\author{
Bo Hu, Zhou Yan, Peng Li, Xu Zhang, Mingxiu Cai, Zhuang Tian, and Panpan Sun \\ School of Engineering Training Center, Shenyang Aerospace University, Shenyang 110136, China.
}

Keywords: intelligent trash can, automatic bag change, infrared sensor cover

Abstract: This paper mainly elaborates the structure and working principle of the automatic bag changing device for trash cans. Incorporating the infrared inductive opening technology of the existing trash can, a new type of automatic household intelligent trash can is designed. The function of automatic closing, bag changing and induction flipping of the trash can is realized, and the intelligent level of the trash can is greatly improved.

\section{Research Background}

Trash cans play an irreplaceable role in today's family life, and now the trash cans on the market are generally manually opened; Garbage bags are hand-stitched when the waste has been filled, which increases the chance of hand-to-bacteria contact and frequent bagging is troublesome. In order to solve the above problems, under the guidance of the people-oriented design concept, the automatic intelligent trash can was designed and manufactured.

\section{The Function and Structure Design of the Trash Can}

\subsection{Overall structure design.}

In the original trash can, the automatic opening of the cover is added, and the function of the garbage bag closing and the automatic bagging is designed. The overall structure of a smart trash can is shown in Figure 1.

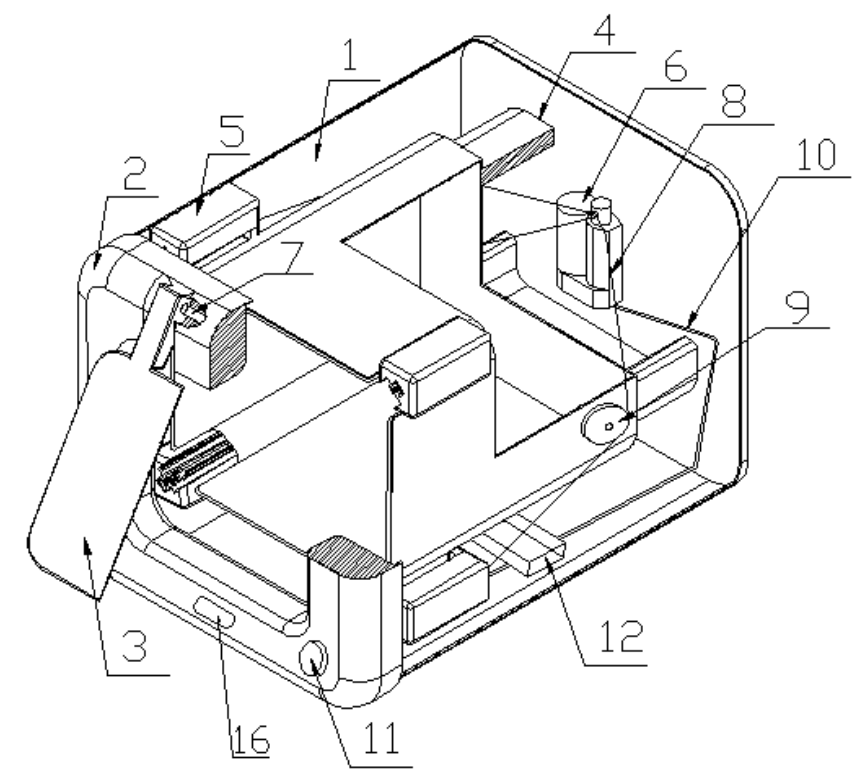




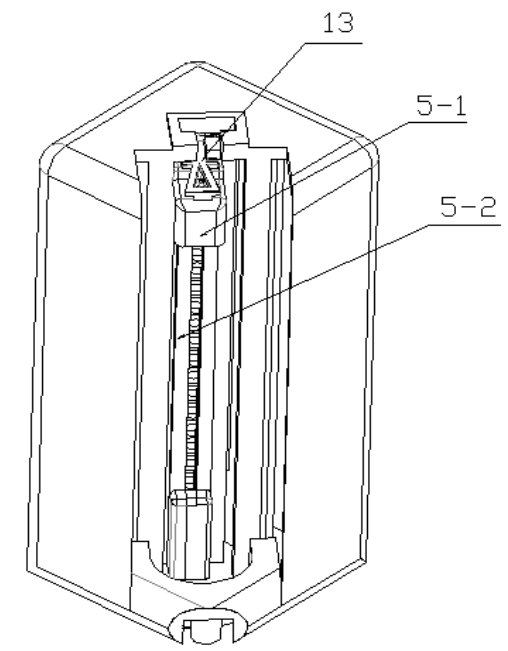

Fig. 1 Overall structure and the holder assembly drawing

1. outer barrel 2. barrel cover 3. flip cover 4. inner barrel 5. the holder 6. Motor 7. Pin 8. Pull wire

9. Guide pulley10. Wire 11. Change bag switch 12. Battery 13. T-type buckle 14. Rubber band 15. Garbage bag 16. Infrared sensing area 5-1. pressure plate 5-2. T- type card slot

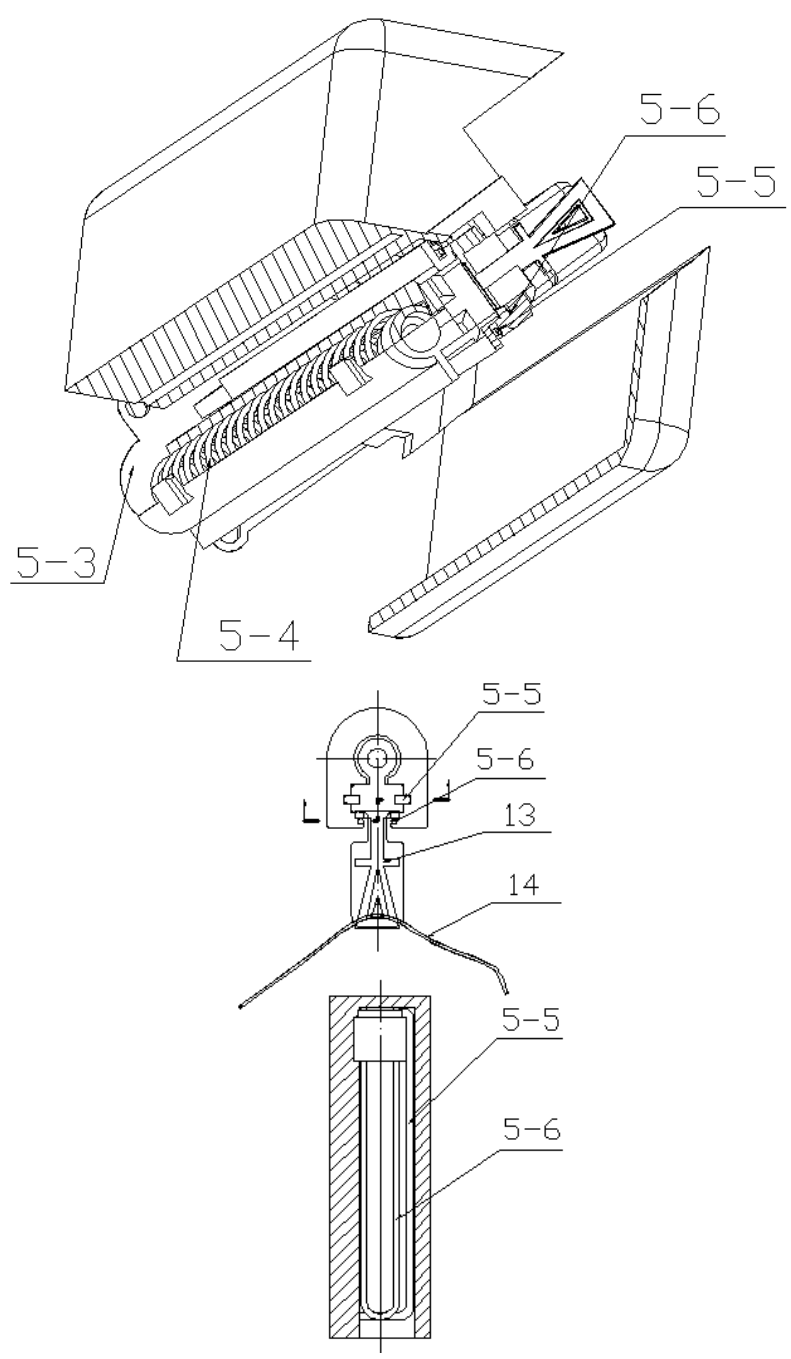

Fig. 2 Cutaway view of the holder

5-3. Plugging plate 5-4. Spring 5-5. Large card strip 5-6. Small card strip 14. Rubber band 


\subsection{The Relationship between the Various Parts.}

The inner bucket 4 is inside the outer bucket 1, and the four upper corners of the inner bucket 4 are fixed with four holders 5 for holding the garbage bag snaps; the holder 5 is connected by the guide pulley 9 and the DC push rod motor 6 is moved by pulling the wire 8 . The motor 6 is fixed at the bottom of the inner and outer barrels. The motor 6 and the battery 12 and the switch form a closed loop through the wire 10, and the battery 12 is fixed to the outer barrel wall. The disassembly and replacement can be performed. The bag change switch 11 is arranged at the bottom right corner of the barrel cover; The cover 2 is covered on top of the entire trash can. The flip cover 3 has an infrared automatic sensing system and is connected to the cover through the pin 7 . The outer tub and the lid form the overall external structure of the trash can, the induction opening device is installed inside the lid to realize the function of opening the lid, the inner barrel is used for holding the garbage bag, and the bag changing device is installed between the inner barrel and the outer barrel.

\subsection{Working Principle.}

The inductive opening and closing device adopts the existing technology_infrared sensor, which is based on the single-chip microcomputer control, realizes infrared distance sensing, and automatically opens and closes the overall structure of the lid. Infrared emission receiving device is used to detect when the objects within the distance from the lid 20CM are blocked, the lid is opened automatically, and the object is automatically closed after leaving the lid.

Each Trash Bag is equipped with four T-type buckles, corresponding to the four corners of the Trash Cartridge. Each T-type card slot can be equipped with 10 T-type buckles at a time. Load 10 garbage bags and wait until the garbage bags are used up before reloading.

When the garbage is full, press the bag change switch, the motor is energized, the motor push rod is extended, and the pull wire is driven. The other end of the pull wire is connected to the size card strips in the four card holders respectively, and the large card strip pulls the pressure plate and moves downwards. At the same time, the small card strip releases the blocking of the T-shaped buckle, and under the tension of the rubber band, the T-shaped buckle is disengaged from the card seat, and the garbage bag mouth is contracted, thereby realizing the sealing of the garbage bag. When the T-type buckle is released from the card holder, the next T-type card buckle is added to complete the work of bag replacement.

When the motor push reaches the maximum value, the bag changing work has been basically completed, and the motor automatically returns. Under the action of the return spring, the pressure plate and the large strip return to the original position, and the U-shaped small strip is pulled in the $\mathrm{V}$-shaped groove. The slack automatically rebounds and the entire system returns to its original state. The bag change system works cyclically.

\section{Innovation and Application}

Through the infrared automatic sensing system, the mechanical structure movement is triggered to realize the function of automatically opening the cover within $20 \mathrm{~cm}$, which avoids the situation that the human hand touches the trash can cover or cannot be manually opened under certain special conditions; When the garbage is full, press the button to trigger the mechanical device to realize the closing of the garbage bag and the replacement of the next garbage bag, avoiding the contact between the hand and the garbage bag, thus preventing the bacteria from communicating through the contact. Prevent human health from being violated; It has the advantages of reliable performance, health and environmental protection, convenient use, simple structure and convenient manufacture.

\section{Conclusion}

This product adds new functions of automatic closing and changing bags on the basis of the original automatic flip trash can, making it more versatile and intelligent; it is superior to similar products in the market in terms of health and environmental protection; It has improved the public 
places and home grades, and is the best choice for office, home, conference, hospital, etc. It makes people's life more concise and convenient, and the market prospect will be very broad.

\section{Acknowledgements}

Fund project: Shenyang Aerospace University Student's Platform for Innovation and Entrepren-eurship Training Program.

First author: Bo Hu (1997 -- ), male, undergraduate, main research direction is aircraft design.

Corresponding author: Zhou Yan (1982 -- ), male, undergraduate, main research direction is the application of numerical control technology and mechanical design research.

\section{References}

[1] C.G. Jia, J.H. Wang: Journal of Jilin Agricultural Science and Technology College, Vol. 20 (2011) No.1, p.49-50.

[2] B.C. Wang, Y. Jiang: Environmental Health Engineering, Vol. 16 (2008) No.5, p.63-64.

[3] L.N. Fan, Z.Q. Bai: Electronic Instrumentation Customer, Vol. 15 (2008) No.6, p.16-17.

[4] T.N. Nie, J. Huang: Journal of Hunan University of Science and Engineering, Vol. 33 (2012) No.4, p.163-166.

[5] C. Zhang: Mechanical Principles and Mechanical Design (Mechanical Industry Press, China 2004).

[6] T. Liang, G.Q. Chang: Mechanical Enginee, Vol. 41 (2009) No.1, p.155.

[7] H.J. Zhou, J.B. Xu: Journal of Guangdong University of Technology, Vol. 23 (2006) No.3, p.85-88. 